\title{
Safety Analysis of Pharmacological Stress Echocardiography with Dipyridamole in Octogenarians
}

\author{
Lucas Munareto Bajerski, José Luis de Castro e Silva Pretto, Raquel Melchior Roman, Eduardo Dal Magro Marcon \\ Hospital São Vicente de Paulo, Passo Fundo, RS - Brazil
}

\section{Summary}

Background: The pharmacological stress echocardiography with dipyridamole is known as safe and accurate test for diagnostic and prognostic investigation of coronary artery disease, particularly useful for elderly who have comorbidities that limit the use of physical stress. Few studies have evaluated the safety of this method in patients over 80 years.

Objective: Evaluate the safety of pharmacological stress echocardiography with dipyridamole in octogenarians.

Methods: A retrospective descriptive study.

Results: The study included 262 patients with a mean age of $82.9 \pm 2.9$ years who underwent a pharmacological stress echocardiogram with dipyridamole $0.84 \mathrm{mg} / \mathrm{kg}$ over 4 minutes. The incidence of complications was $3.4 \%$ (nine cases), only one major complication $(0.4 \%)$, which was a case of prolonged ischemia requiring urgent invasive treatment. Other complications were two cases of prolonged ischemia treated with beta blocker, three cases of transient supraventricular tachycardias, one case of sustained supraventricular tachycardia reversed with adenosine, one case of atrial fibrillation and one case of transitory atrioventricular block 2:1.

Conclusion: In this study the stress echocardiography with dipyridamole was shown to be a safe test in the selected population of octogenarians. (Arq Bras Cardiol: Imagem cardiovasc. 2016;29(3):80-83)

Keywords: Coronary Artery Disease; Echocardiography, Stress; Dipyridamole; Aged 80 and Over; Patient Safety.

\section{Introduction}

Pharmacological stress echocardiography using dipyridamole is recognized as an accurate and safe test for diagnostic and prognostic investigation of coronary artery disease. ${ }^{1}$ It is a relatively accessible tool that is especially useful in individuals with advanced age, who have higher prevalence of musculoskeletal, vascular and neurological conditions that limit the use of physical stress.

Dipyridamole is a coronary vasodilator and its effects result from inhibition of adenosine uptake. The development of abnormalities in the test is based on the ability to cause heterogeneous distribution of regional blood flow and consequent abnormalities in systolic movement and thickening of the ventricular walls induced by ischemia. ${ }^{2}$

The test is known to be safe in the general population. The international registration of the Echo-Persantine International Cooperative Study Group showed a prevalence

Mailing Address: Residência de Ecocardiografia - Hospital São Vicente de Paulo •

Rua Teixeira Soares, 808. Postal Code 99010-080, Centro, Passo Fundo, RS - Brazil

Email: lucasmunareto@yahoo.com.br

Manuscript received December 18, 2015; revised January 13, 2016; accepted June 06, 2016.

DOI: $10.5935 / 2318-8219.20160022$ of major complications (acute pulmonary edema, ventricular tachycardia, myocardial infarction and cardiac arrest in asystole) of $0.07 \%$ in patients aged 24 to 80 . Other minor complications were tachyarrhythmia $(0.1 \%)$, hypotension and bradyarrhythmia $(0.5 \%){ }^{3}$

However, there are few studies evaluating the safety of stress echocardiography with dipyridamole in individuals aged over 80, which increasingly represent our population.

The objective of this study is to evaluate the safety of pharmacological stress echocardiography with dipyridamole in octogenarians.

\section{Methodology}

A retrospective descriptive study included patients older than 80, who underwent pharmacological stress echocardiography with dipyridamole from 2004 to 2015, recorded in the database of the echocardiography laboratory of a tertiary hospital.

As a routine of the health service, the patients were advised not to smoke and to avoid using xanthine derivatives such as tea, coffee or soft drinks for 24 hours before the test. The patients underwent a clinical interview and had resting electrocardiogram and echocardiogram before the stress protocol.

Demographic data and clinical history were collected (symptoms, cardiovascular risk factors, history of coronary artery disease, previous coronary artery bypass grafting, 
treatments in course and test indications). Second or third degree atrioventricular block, sick sinus syndrome, asthma and bronchospasm are contraindications to stress with dipyridamole. ${ }^{4}$ For atropine, the main contraindications are glaucoma and prostatism. ${ }^{5}$

The standard pharmacological stress protocol was a continuous intravenous infusion of dipyridamole at $0.84 \mathrm{mg} / \mathrm{kg}$ for 4 minutes. Atropine was used at a dose of $1.0 \mathrm{mg}$ and/or hand grip maneuver at the discretion of the doctor running the test in the $6^{\text {th }}$ minute. At the end of the test, aminophylline was used in all patients in the $10^{\text {th }}$ minute. ${ }^{6}$

Coronary flow reserve (CFR) in the anterior descending coronary artery is part of the standard protocol of the institution. CFR is estimated by the ratio between the peak diastolic velocity in hyperemia induced by dipyridamole and at rest. CFR is considered low when it is smaller than 2 and adds independent prognostic value of segmental contractility analysis. ${ }^{7,8}$

Analysis was conducted in 16 segments and five possible answers according to the segmental contractility: normal, hypokinesia, akinesia, dyskinesia and aneurysm. ${ }^{9}$ The test was considered positive for ischemia when there was a new segment impairment or additional in a previously hypokinetic segment.

Recommendations for test interruption were: ischemia in two or more left ventricular segments, especially in the presence of cavity dilatation or reduced ejection fraction; occurrence of advanced and persistent atrioventricular block; another complication or significant side effect. ${ }^{10}$

In assessing the safety outcomes, major complications were: acute pulmonary edema, tachycardia or ventricular fibrillation, acute myocardial infarction, or urgent coronary artery bypass grafting, cardiac arrest and death. As minor complications, we considered prolonged ischemia (defined as persistent ischemia after using aminophylline), symptomatic bronchospasm, bradyarrhythmia and tachyarrhythmia.

\section{Study population}

The records of 262 patients aged 82.8 ( \pm 2.9 years) were analyzed. Of these patients, $56 \%$ were females.

All tests were performed to evaluate ischemia and in $29 \%$ of patients, the investigation was part of the preoperative risk assessment. Regarding the symptoms, $43 \%$ of the patients had dyspnea, $34 \%$ had atypical pain, $18 \%$ had typical pain and $4 \%$ had syncope.

Regarding the risk factors, the prevalence of hypertension was $87 \%$, dyslipidemia $55 \%$, diabetes $23 \%$ and current smoking habits $1 \%$. In this population, $28 \%$ of the patients had known coronary artery disease, $22 \%$, contractility abnormalities at rest and $10 \%$, acute myocardial infarction, and $18 \%$ had undergone percutaneous coronary angioplasty and $2 \%$, coronary artery bypass grafting (Table 1 ).

As for the medications, 56\% were using statins, 53\% used diuretics, 49\% used acetylsalicylic acid (AAS), 13\% used clopidogrel, 44\% used beta blockers, 36\% were using angiotensin receptor blockers and 33\% used angiotensinconverting enzyme inhibitors. Of the patients, $18 \%$ had contraindications to the use of atropine.
Table 1 - Characteristics of the study population (\%)

\begin{tabular}{lc}
\hline Mean age & $\mathbf{8 2 . 8}$ years \\
\hline Women & 56 \\
Evaluation of ischemia & 100 \\
Pre-operative evaluation & 29 \\
Dyspnea & 43 \\
Atypical chest pain & 34 \\
Typical chest pain & 18 \\
Syncope & 4 \\
Hypertension & 87 \\
Dyslipidemia & 55 \\
Diabetes & 23 \\
Coronary artery disease & 28 \\
Acute myocardial infarction & 10 \\
\hline
\end{tabular}

\section{Results}

The test was positive for ischemia in $24 \%$ of the cases; in $6 \%$, there was reduced ejection fraction; and in 3\%, the test was discontinued earlier for positivity. The flow reserve in the anterior descending artery was assessed in 215 patients $(82 \%)$ and in $38 \%$ of the cases, it was reduced. Atropin was used in $52 \%$ of cases.

The incidence of complications in the study sample was $3.4 \%$ (9 cases), with only one major complication $(0.4 \%)$.

The major complication was a case of prolonged ischemia requiring urgent invasive treatment. In this case, the patient had mild hypokinesia in the segments anterior medial and septum apical at rest. On stress, the test was discontinued earlier for positivity in low dose, with akinesia in the segments inferior apical, anterior medial and apical, anterior septum medial and septum apical, with a drop in ejection fraction. Because the patient continued with chest pain despite the medical therapy established, the patient was referred for emergency coronary angiography, which revealed total occlusion of the medial anterior descending and proximal circumflex arteries; the patient also presented mild collateral circulation to the distal anterior descending artery. Angioplasty was performed with the implantation of three stents in the circumflex artery; it was not possible to run the guide wire due to obstruction of the anterior descending artery, considered as probably chronic. There was improvement in the patient's symptoms. Resting echocardiogram was conducted on the day following the event, showing hypokinesia in the inferior apical and septum apical segments.

The other complications were: Two cases of prolonged ischemia treated with intravenous beta-blockers (metoprolol) and 6 cases of arrhythmia. Among these, there was one 2:1 transient atrioventricular block and 3 transient supraventricular tachycardia events. One patient had sustained supraventricular tachycardia reversed with adenosine and another one had atrial fibrillation and was referred for the hospital's emergency department. 
The main symptoms caused by the test were headache $(14 \%)$, chest pain $(9 \%)$, palpitations $(7 \%)$, dyspnea $(6 \%)$ and nausea (2\%). The test was discontinued earlier due to symptoms only in one case, due to nausea.

\section{Discussion}

With the progressive increase in the population's life expectancy, we need accurate non-invasive tools to establish diagnosis and prognosis of ischemic heart disease in old age patients.

The safety of stress echocardiography using dipyridamole in accelerated protocol and atropine was also demonstrated in an Italian study with 337 patients with good tolerance and no major complications. ${ }^{6}$ Similarly, another group of researchers using dipyridamole stress protocol similar to our study found that the test is feasible and safe in a publication including 752 patients in which wall motility and coronary flow reserve were simultaneously analyzed.

As expected, we observed in this sample a high prevalence of risk factors and known coronary artery disease, which could result in a significant increase in major complications, but this was not observed.

In line with literature data on the general population, we found a low incidence of major complications in a selected group of patients aged over 80 years, which was only 1 case $(0.4 \%)$, due to severe persistent ischemia.

In the general population, the international registration of the Echo-Persantine International Cooperative Study Group ${ }^{4}$, evaluating 9,122 patients aged 24 to 80, found 7 cases of major complications $(0.07 \%), 6$ of which are related to ischemia. This study revealed that in these 6 cases, the professional doing the test was still learning, showing that experience in performing the dipyridamole stress echocardiography is connected with the safety of the method.

Analyzing the characteristics of the subgroup with complications (Table 2), we observed that more patients presented abnormalities in contractility at rest, the use of atropine was larger and more tests were positive for ischemia. The association between the use of atropine and the presence of ischemia with the occurrence of complications has also been found in previous studies. . $^{41,12}$

\section{Limitations}

This study has some limitations related to the relatively small sample size and retrospective design, which may have resulted in underreporting of minor symptoms.
Table 2 - Characteristics of the subgroup with complications (\%)

\begin{tabular}{lc}
\hline Mean age & $\mathbf{8 2 . 4}$ years \\
\hline Women & 67 \\
Hypertension & 87 \\
Dyslipidemia & 50 \\
Diabetes & 13 \\
Coronary artery disease & 13 \\
Contractility abnormalities & 33 \\
Use of atropin & 78 \\
Positive for ischemia & 44 \\
\hline
\end{tabular}

"at rest

\section{Conclusion}

Although it is a small sample, we can conclude that stress echocardiography with dipyridamole was a safe test in the selected group of octogenarians.

\section{Authors' contributions}

Research creation and design: Bajerski LM, Pretto JLCS, Roman RM, Marcon EDM; Data acquisition: Bajerski LM, Marcon EDM; Data analysis and interpretation: Bajerski LM, Pretto JLCS, Roman RM, Marcon EDM; Statistical analysis: Bajerski LM, Roman RM; Manuscript drafting: Bajerski LM, Pretto JLCS, Roman RM, Marcon EDM; Critical revision of the manuscript as for important intellectual content: Bajerski LM, Pretto JLCS, Roman RM, Marcon EDM.

\section{Potential Conflicts of Interest}

There are no relevant conflicts of interest.

\section{Sources of Funding}

This study had no external funding sources.

\section{Academic Association}

This study is not associated with any graduate program. 


\section{References}

1. Picano E. Stress echocardiography. Minerva Cardioangiol. 2005; 53(3):195-210.

2. Armstrong FH, Ryan T. Feigenbaum's echocardiography. 7th ed. Philadelphia: Lippincott Williams and Wilkins; 2010. p. 465-98

3. Picano E, Marini C, Pirelli S, Maffei S, Bolognese L, Chiriatti G, et al. Safety of intravenous high-dose dipyridamole echocardiography. Am J Cardiol.1992;70(2):252-8.

4. Picano E. Stress echocardiography. $5^{\text {th }}$ ed. Pisa: Springer-Verlag ; 2009. p. 189-206.

5. Picano E. Stress echocardiography. $5^{\text {th }}$ ed. Pisa: Springer-Verlag ; 2009. p. 57-73.

6. Minardi G, Manzara CC, Pulignano G,Carmenini E, Gaudio C, Giovannini E. Safety and diagnostic accuracy of intravenous accelerated high-dose dipyridamole-atropine stress echocardiography. Ital HeartJ. 2002;3(12):726-9.

7. Lowenstein J, Tiano C, Marquez G, Pesti C, Quiroz C. Simultaneous analysis of wall motion and coronary flow reserve of the left anterior descending coronary artery by transthoracic Doppler echocardiography during dipyridamole stress echocardiography. J Am Soc Echocardiogr. 2003;16(6):607-13.
8. Cortigiani L, Rigo F, Gherardi S, Sicari R, Galderisi M, Bovenzi $\mathrm{F}$, et al. Additional prognostic value of coronary flow reserve in diabetic and nondiabetic patients with negative dipyridamole stress echocardiography by wall motion criteria. J Am Coll Cardiol. 2007;50(14):1354-61.

9. Schiller N, Shah P, Crawford M, DeMaria A, Devereux R, Feigenbaum H, et al. Recommendations for quantitation of left ventricle by two-dimensional echocardiography. J Am Soc Echocardiogr. 1989;2(5):358-67.

10. Pellikka PA, Nagueh SF, Elhendy AA, Kuehl CA, Sawada SG . American Society of Echocardiography recommendations for performance, interpretation and application of stress echocardiography. J Am Soc Echocardiogr. 2007;20(9):1021-41.

11. Nedeljkovic MA, Ostojic M, Beleslin B, Nedelykovic IP, Stankovic G, Stojkovic S, et al. Dipyridamole-atropine-induced myocardial infarction in a patient with patent epicardial coronary arteries. Herz. 2001;26(7):485-8.

12. Erdogan O, Altun A, Akdemir O, Aktoz M, Ozbaya G. Unexpected occurrence of ST segment elevation during administration of intravenous atropine. Cardiovasc Drugs Ther. 2001;15(4):367-8. 